\title{
MICROWAVE-ASSISTED SYNTHESIS OF BENZOFURAN ANALOGS OF FENAMATES AS NON STEROIDAL ANTI-INFLAMMATORY AGENTS
}

\author{
BALASAHEB Y. MANE ${ }^{1}$, Y.S.AGASIMUNDIN², B. SHIVKUMAR², DEVANAND B. SHINDE ${ }^{3 *}$ \\ ${ }^{1}$ SVPM's College of Pharmacy, Malegaon BkII, Baramati, Dist- Pune-413115 (M.S.) India. \\ ${ }^{2}$ Dept. of Pharmaceutical Chemistry, S.C.S. College of Pharmacy Harpanahalli, Dist- Davanageere-583131, Karnataka, India. \\ ${ }^{*}$ Department of Chemical Technology, Dr. BabasahebAmbedkar Marathwada University, Aurangabad-431004 (M.S.) India.
}

(Received 27 June 2008 - Accepted 26 November 2008)

\begin{abstract}
A series of benzofuran analogs of anthranilic acid derivatives were easily synthesized by microwave irradiation and conventional heating method. The microwave irradiation method gives the comparable yield as that of conventional heating with a shorter reaction time. All the new compounds have been characterized by spectral data and screened for anti-inflammatory activity.
\end{abstract}

Key words: benzofuran, anthranilic acid, anti-inflammatory activity, microwave irradiation.

\section{INTRODUCTION}

The application of microwave as an energy source for chemical reactions and processes has been extensively studied during recent years'. The combination of solvent-free conditions and microwave irradiation leads to large reduction in reaction time, enhancement in conversion, easier work up and sometimes in selectivity with several advantages of an ecofriendly approach ${ }^{2}$. Further, its unique capabilities allow its applications in transformations which are difficult or impossible to carry out by means of conventional method ${ }^{3}$.

The fenamates are the class of the NSAIDs that have common structural features of an $\mathrm{N}$-arylanthranilic acid such as mefenamic acid, meclofenamic acid and flufenamic acid ${ }^{4-6}$. These are the first line therapeutic agents for the clinical treatment of various inflammatory disorders ${ }^{7}$. In view of this several anthranilic acid derivatives have been prepared for investigation of antiinflammatory activity. Many such compounds were found to possess potent anti-inflammatory ${ }^{8-10}$, cardiovascular $^{11}$ and analgesic ${ }^{12}$ activites. Anthranilic acid derivatives are known to act by blocking the metabolism of arachidonic acid by inhibiting the enzyme cyclooxygenase $(\mathrm{CO})$ and thereby the production of prostaglandins. Inhibiting $\mathrm{CO}$ may also increase the conversion of arachidonic acid to proinflammatory leukotrienes via the enzyme 5-Lipoxygenase (5-LO). Literature survey shows that modification of carboxylic group in anthranilic acid markedly modulates the activity. The modification of the carboxylic acid functionality not only retained the $\mathrm{CO}$ inhibitory activity of the parent but also inhibited 5- $\mathrm{LO}^{13-14}$.

Compounds containing benzofuran nucleus are widely distributed in nature amongst the plant kingdom. Such compounds are often associated with useful biological activities. This stimulated our interest in the synthesis of benzofuran analogs of anthranilic acid derivatives in which the benzene moiety of anthranilic acid is replaced by benzofuran moiety.

Ethyl 3-hydroxybenzofuran-2-carboxylate was prepared by single pot synthesis ${ }^{15}$ from methyl salicylate and diethylbromo malonate served as key starting material for the synthesis of target molecules. The reaction of this with various aromatic amines in presence of catalytic amount of hydrochloric acid produced ethyl-3-arylaminobenzofuran-2-carboxylates. This needs heating of the reaction mixture for $90-95^{\circ} \mathrm{C}$ for 9 hrs. This is a very time consuming reaction. The same reaction mixture when irradiated in microwave for different period of time at $500 \mathrm{~W}$ power gives the desired product. As compared to conventional heating method the microwave irradiation method for synthesis of ethyl -3-arylaminobenzofuran-2-carboxylates is simple and less time consuming. As this reaction does not involve use of any solvents so there is no any risk of explosion of the solvents during microwave irradiation.

\section{EXPERIMENTAL}

Melting points were determined in open capillary tubes and are uncorrected. The FT-IR spectra were recorded in $\mathrm{KBr}$ on SHIMADZU FTIR-8400S spectrophotometer. ${ }^{1} \mathrm{H}$ NMR spectra were recorded in $\mathrm{CDCI}_{3}$ on a Varian Mercury YH-300, using TMS as an internal standard. Microwave irradiations were carried out in a LG intellocook MS-1921HE with RF output of 700W.

\section{SCHEME}

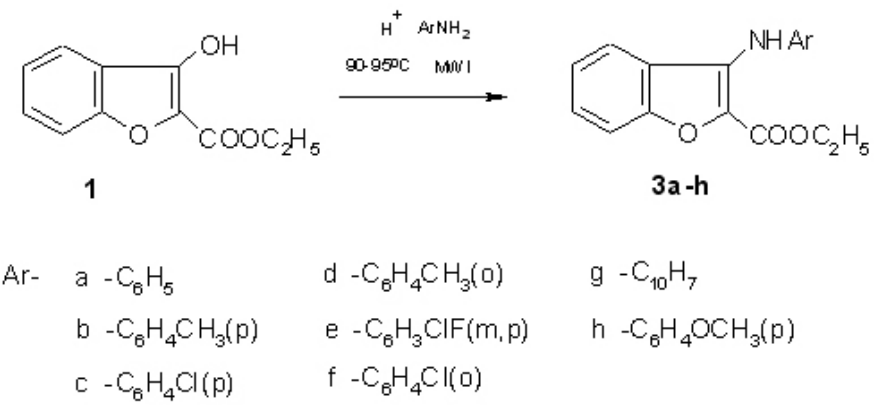

Preparation of Ethyl 3-arylaminobenzofuran-2-carboxylates (3a-h) by conventional heating method:

An intimate mixture of $\mathbf{1}(2.0 \mathrm{~g}, 0.01 \mathrm{~mol})$, aromatic amine $(0.011$ mol) and 3-4 drops of concd. hydrochloric acid was left overnight at room temperature and then heated at $90-95^{\circ} \mathrm{C}$ for $9 \mathrm{hr}$. The reaction mixture was cooled and extracted with ether $(3 \times 20 \mathrm{ml})$. The combined organic layer was washed successively with $2 \mathrm{~N}$ hydrochloric acid and aqueous sodium hydroxide $(10 \%)$ to remove the unreacted starting materials, dried over anhydrous sodium sulphate. Removal of the solvent by evaporation gave the product, which was crystallized from aqueous ethanol.

Preparation of Ethyl 3-arylaminobenzofuran-2-carboxylates (3a-h) by Microwave irradiation method:

An intimate mixture of $\mathbf{1}(2.0 \mathrm{~g}, 0.01 \mathrm{~mol})$, aromatic amine $(0.011 \mathrm{~mol})$ and a 3-4 drop of concd. hydrochloric acid was transferred to the conical flask. The reaction mixture was subjected to Microwave irradiation for different time period as mentioned in table No-1 under $500 \mathrm{~W}$ power. Then reaction mixture was cooled and extracted with ether $(3 \times 20 \mathrm{ml})$. The combined organic layer was washed successively with $2 \mathrm{~N}$ hydrochloric acid and aqueous sodium hydroxide $(10 \%)$ to remove the unreacted starting materials and dried over anhydrous sodium sulphate. Removal of the solvent by evaporation gave the product, which was crystallized from aqueous ethanol. The characterization data is given in Table No-1 and Table No- 2.

\section{BIOLOGICAL ACTIVITY ${ }^{16-18}$}

All the compounds were screened for anti-inflammatory activity by carrageenan induced rat paw edema method. Diclofenac sodium was used as standard. Rats were divided into control, standard and different test groups comprising of six animals in each group. They were fasted overnight with free access to water before experiment. In all groups acute inflammation was induced by subplantar injection of $0.1 \mathrm{ml}$ of freshly prepared $1 \%$ suspension of carrageenan in the right hind paw of the rats and paw volume was measured Plethysmometrically at 0 hour and $3 \mathrm{hr}$ after carrageenan injection. Rats of test groups were administered orally with test compounds $100 \mathrm{mg} / \mathrm{kg}$ and the standard group with diclofenac $100 \mathrm{mg} / \mathrm{kg}$ orally in $2 \%$ aqueous acacia one $\mathrm{hr}$ 
before injection of carrageenan. Control group received only vehicle. Mean difference in paw volume was measured and percentage of inhibition of edema was calculated and given in Table No-4.

Table No- 1. Physical, analytical data of the compounds 3a-h

\begin{tabular}{|c|c|c|c|c|c|c|}
\hline Compound & $\begin{array}{c}\text { MP } \\
{ }^{0} \mathbf{C}\end{array}$ & $\begin{array}{c}\text { Yield } \\
\mathbf{( \% )} \\
\text { Heating }\end{array}$ & $\begin{array}{c}\text { Yield } \\
\mathbf{( \% )} \\
\mathbf{M W I}\end{array}$ & $\begin{array}{c}\text { Power } \\
\mathbf{W}\end{array}$ & $\begin{array}{c}\text { Time } \\
\text { Heating } \\
\text { Min }\end{array}$ & $\begin{array}{c}\text { Time } \\
\text { MWI } \\
\text { Min }\end{array}$ \\
\hline 3a & 84 & 15 & 3 & 500 & 540 & 2 \\
\hline $3 \mathrm{~b}$ & 128 & 27 & 27 & 500 & 540 & 2 \\
\hline $3 \mathrm{c}$ & 125 & 63 & 70 & 500 & 540 & 2 \\
\hline $3 \mathrm{~d}$ & 98 & 23 & 20 & 500 & 540 & 4 \\
\hline $3 \mathrm{e}$ & 103 & 34 & 45 & 500 & 540 & 4 \\
\hline $3 \mathrm{f}$ & 111 & 15 & 13 & 500 & 540 & 5 \\
\hline $3 \mathrm{~g}$ & 98 & 33 & 25 & 500 & 540 & 5 \\
\hline $3 \mathrm{~h}$ & 102 & 64 & 57 & 500 & 540 & 4 \\
\hline
\end{tabular}

All the compounds were recrystallised from aqueous ethanol.

Table No-2. Elemental analysis data for the compounds 3a-h

\begin{tabular}{|c|c|c|c|c|c|}
\hline \multirow{2}{*}{ Sr. No } & \multirow{2}{*}{ Compounds } & \multirow{2}{*}{$\begin{array}{l}\text { Molecular } \\
\text { formula }\end{array}$} & \multicolumn{3}{|c|}{$\begin{array}{c}\text { Elemental analysis } \\
\text { Found (calculated) }(\%)\end{array}$} \\
\hline & & & C & $\mathbf{H}$ & $\mathbf{N}$ \\
\hline 01 & $3 a$ & $\mathrm{C}_{17} \mathrm{H}_{15} \mathrm{NO}_{3}$ & $\begin{array}{c}72.50 \\
(72.59)\end{array}$ & $\begin{array}{c}5.25 \\
(5.33)\end{array}$ & $\begin{array}{c}4.78 \\
(4.98)\end{array}$ \\
\hline 02 & $3 b$ & $\mathrm{C}_{18} \mathrm{H}_{17} \mathrm{NO}_{3}$ & $\begin{array}{c}73.05 \\
(73.22)\end{array}$ & $\begin{array}{c}5.62 \\
(5.76)\end{array}$ & $\begin{array}{c}4.61 \\
(4.74)\end{array}$ \\
\hline 03 & $3 c$ & $\mathrm{C}_{17} \mathrm{H}_{14} \mathrm{NO}_{3} \mathrm{Cl}$ & $\begin{array}{l}64.55 \\
(64.65)\end{array}$ & $\begin{array}{c}4.35 \\
(4.44)\end{array}$ & $\begin{array}{c}4.32 \\
(4.44)\end{array}$ \\
\hline 04 & $3 d$ & $\mathrm{C}_{18} \mathrm{H}_{17} \mathrm{NO}_{3}$ & $\begin{array}{c}73.20 \\
(73.22)\end{array}$ & $\begin{array}{c}4.52 \\
(4.74)\end{array}$ & $\begin{array}{c}4.58 \\
(4.74)\end{array}$ \\
\hline 05 & $3 e$ & $\mathrm{C}_{17} \mathrm{H}_{13} \mathrm{NO}_{3} \mathrm{ClF}$ & $\begin{array}{c}51.70 \\
(51.71)\end{array}$ & $\begin{array}{c}3.12 \\
(3.20)\end{array}$ & $\begin{array}{c}4.02 \\
(4.20)\end{array}$ \\
\hline 06 & $3 \mathrm{f}$ & $\mathrm{C}_{17} \mathrm{H}_{14} \mathrm{NO}_{3} \mathrm{Cl}$ & $\begin{array}{c}64.56 \\
(64.65)\end{array}$ & $\begin{array}{c}4.30 \\
(4.44)\end{array}$ & $\begin{array}{c}4.31 \\
(4.44)\end{array}$ \\
\hline 07 & $3 g$ & $\mathrm{C}_{21} \mathrm{H}_{17} \mathrm{NO}_{3}$ & $\begin{array}{c}76.05 \\
(76.13)\end{array}$ & $\begin{array}{c}5.10 \\
(5.13)\end{array}$ & $\begin{array}{c}4.05 \\
(4.22)\end{array}$ \\
\hline 08 & $3 \mathrm{~h}$ & $\mathrm{C}_{18} \mathrm{H}_{17} \mathrm{NO}_{4}$ & $\begin{array}{c}69.32 \\
(69.45)\end{array}$ & $\begin{array}{c}5.34 \\
(5.40)\end{array}$ & $\begin{array}{c}4.37 \\
(4.50)\end{array}$ \\
\hline
\end{tabular}

Table No- 3. Spectral data of the Compounds 3a-h

\begin{tabular}{|c|c|c|}
\hline Compound & IR & ${ }^{1} \mathrm{H}$ NMR \\
\hline $3 a$ & $\begin{array}{l}3360,3440(\mathrm{NH}), \\
1685(\mathrm{C}=\mathrm{O}), \\
1610(\mathrm{C}=\mathrm{N}), 1575(\mathrm{C}=\mathrm{C}), \\
1100-1120(\mathrm{C}-\mathrm{O}-\mathrm{C})\end{array}$ & $\begin{array}{l}1.5\left(\mathrm{t}, 3 \mathrm{H}, \mathrm{CH}_{2} \mathrm{CH}_{3}, J=7.1\right. \\
\mathrm{Hz}), 1.6(\mathrm{~s}, 1 \mathrm{H}, \mathrm{NH}), 4.5(\mathrm{q}, \\
\left.2 \mathrm{H}, \mathrm{CH}_{2} \mathrm{CH}_{3}, J=7.1 \mathrm{~Hz}\right), \\
7-7.8(\mathrm{~m}, 9 \mathrm{H}, \mathrm{ArH},)\end{array}$ \\
\hline $3 b$ & $\begin{array}{l}3350,3450(\mathrm{NH}), 1680 \\
(\mathrm{C}=\mathrm{O}), \\
1620(\mathrm{C}=\mathrm{N}), 1580(\mathrm{C}=\mathrm{C}), \\
1095,1120(\mathrm{C}-\mathrm{O}-\mathrm{C})\end{array}$ & $\begin{array}{l}1.5\left(\mathrm{t}, 3 \mathrm{H}, \mathrm{CH}_{2} \mathrm{CH}_{3}, J=7.1\right. \\
\mathrm{Hz}), 1.6(\mathrm{~s}, 1 \mathrm{H}, \mathrm{NH}), 2.4 \\
\left(\mathrm{~s}, 3 \mathrm{H}, \mathrm{ArCH}_{3}\right), 4.5(\mathrm{q}, 2 \mathrm{H}, \\
\left.\mathrm{CH}_{2} \mathrm{CH}_{3}, J=7.1 \mathrm{~Hz}\right), 7-7.8 \\
(\mathrm{~m}, 8 \mathrm{H}, \mathrm{ArH})\end{array}$ \\
\hline $3 c$ & $\begin{array}{l}3350(\mathrm{NH}), \\
1685(\mathrm{C}=\mathrm{O}), 1610(\mathrm{C}=\mathrm{N}), \\
1575(\mathrm{C}=\mathrm{C}), 1100-1120(\mathrm{C}- \\
\mathrm{O}-\mathrm{C})\end{array}$ & $\begin{array}{l}1.4\left(\mathrm{t}, 3 \mathrm{H}, \mathrm{CH}_{2} \mathrm{CH}_{3} J=7.1\right. \\
\mathrm{Hz}), 1.7(\mathrm{~s}, 1 \mathrm{H}, \mathrm{NH}), 4.4(\mathrm{q}, \\
\left.2 \mathrm{H}, \mathrm{CH}_{2} \mathrm{CH}_{3}, J=7.1 \mathrm{~Hz}\right), \\
7.1-7.8(\mathrm{~m}, 8 \mathrm{H}, \mathrm{ArH})\end{array}$ \\
\hline $3 d$ & $\begin{array}{l}3190,3315(\mathrm{NH}), \\
1670(\mathrm{C}=\mathrm{O}), \\
1600(\mathrm{C}=\mathrm{N}), 1580(\mathrm{C}=\mathrm{C}), \\
1095,1120(\mathrm{C}-\mathrm{O}-\mathrm{C})\end{array}$ & $\begin{array}{l}1.6\left(\mathrm{t}, 3 \mathrm{H}, \mathrm{CH}_{2} \mathrm{CH}_{3}, J=7.1\right. \\
\mathrm{Hz}), 1.6(\mathrm{~s}, 1 \mathrm{H}, \mathrm{NH}), 2.4 \\
\left(\mathrm{~s}, 3 \mathrm{H}, \mathrm{ArCH}_{3}\right), 4.5(\mathrm{q}, 2 \mathrm{H}, \\
\left.\mathrm{CH}_{2} \mathrm{CH}_{3}, J=7.1 \mathrm{~Hz}\right), 7-7.8 \\
(\mathrm{~m}, 8 \mathrm{H}, \mathrm{ArH})\end{array}$ \\
\hline $3 e$ & $\begin{array}{l}3359(\mathrm{NH}), 1666(\mathrm{C}=\mathrm{O}), \\
1604(\mathrm{C}=\mathrm{N}), \\
1577(\mathrm{C}=\mathrm{C}), 1101(\mathrm{C}-\mathrm{O}-\mathrm{C})\end{array}$ & $\begin{array}{l}1.4\left(\mathrm{t}, 3 \mathrm{H}, \mathrm{CH}_{2} \mathrm{CH}_{3}, J=7.1\right. \\
\mathrm{Hz}), 1.6(\mathrm{~s}, 1 \mathrm{H}, \mathrm{NH}), 4.4(\mathrm{q}, \\
\left.2 \mathrm{H}, \mathrm{CH}_{2} \mathrm{CH}_{3}, J=7.1 \mathrm{~Hz}\right), \\
7-7.8(\mathrm{~m}, 7 \mathrm{H}, \mathrm{ArH})\end{array}$ \\
\hline $3 \mathrm{f}$ & $\begin{array}{l}3355(\mathrm{NH}), 1670(\mathrm{C}=\mathrm{O}), \\
1610(\mathrm{C}=\mathrm{N}), \\
1580(\mathrm{C}=\mathrm{C}), 1105(\mathrm{C}-\mathrm{O}-\mathrm{C})\end{array}$ & $\begin{array}{l}1.4\left(\mathrm{t}, 3 \mathrm{H}, \mathrm{CH}_{2} \mathrm{CH}_{3}, J=7.1\right. \\
\mathrm{Hz}), 1.7(\mathrm{~s}, 1 \mathrm{H}, \mathrm{NH}), 4.4(\mathrm{q}, \\
\left.2 \mathrm{H}, \mathrm{CH}_{2} \mathrm{CH}_{3}, J=7.1 \mathrm{~Hz}\right), \\
7.1-7.8(\mathrm{~m}, 8 \mathrm{H}, \mathrm{ArH})\end{array}$ \\
\hline $3 g$ & $\begin{array}{l}3350(\mathrm{NH}), 1680(\mathrm{C}=\mathrm{O}), \\
1610(\mathrm{C}=\mathrm{N}), \\
1575(\mathrm{C}=\mathrm{C}), 1100, \\
1120(\mathrm{C}-\mathrm{O}-\mathrm{C})\end{array}$ & $\begin{array}{l}1.6\left(\mathrm{t}, 3 \mathrm{H}, \mathrm{CH}_{2} \mathrm{CH}_{3}, J=7.1\right. \\
\mathrm{Hz}), 1.5(\mathrm{~s}, 1 \mathrm{H}, \mathrm{NH}), 4.5(\mathrm{q}, \\
\left.2 \mathrm{H}, \mathrm{CH}_{2} \mathrm{CH}_{3}, J=7.1 \mathrm{~Hz}\right), \\
7-7.8(\mathrm{~m}, 9 \mathrm{H}, \mathrm{ArH})\end{array}$ \\
\hline $3 \mathrm{~h}$ & $\begin{array}{l}3355(\mathrm{NH}), 1670(\mathrm{C}=\mathrm{O}), \\
1605(\mathrm{C}=\mathrm{N}), \\
1570(\mathrm{C}=\mathrm{C}), 1100, \\
1125(\mathrm{C}-\mathrm{O}-\mathrm{C})\end{array}$ & $\begin{array}{l}1.5\left(\mathrm{t}, 3 \mathrm{H}, \mathrm{CH}_{2} \mathrm{CH}_{3}, J=7.1\right. \\
\mathrm{Hz}), 1.7(\mathrm{~s}, 1 \mathrm{H}, \mathrm{NH}), 3.9 \\
\left(\mathrm{~s}, 3 \mathrm{H}, \mathrm{OCH}_{3}\right), 4.5(\mathrm{q}, 2 \mathrm{H}, \\
\left.\mathrm{CH}_{2} \mathrm{CH}_{3}, J=7.1 \mathrm{~Hz}\right), 6.9-7.7 \\
(\mathrm{~m}, 11 \mathrm{H}, \mathrm{ArH})\end{array}$ \\
\hline
\end{tabular}

Table No- 4. Biological activity data of the compounds 3a-h

\begin{tabular}{|c|c|c|c|c|}
\hline Sr.No. & Compounds & $\begin{array}{c}\text { Mean } \\
\text { Dose } \\
\mathbf{m g} / \mathbf{k g}\end{array}$ & $\begin{array}{c}\text { difference in } \\
\text { paw volume+ } \\
\text { S.E after 3 } \\
\text { hrs(ml) }\end{array}$ & $\begin{array}{c}\text { Percentage } \\
\text { of inhibition }\end{array}$ \\
\hline 01 & Control & - & $0.90 \pm 0.03^{*}$ & - \\
\hline 02 & Diclofenac & 100 & $0.14 \pm 0.01^{*}$ & 84 \\
\hline 03 & $3 \mathrm{a}$ & 100 & $0.37 \pm 0.02^{*}$ & 58 \\
\hline 04 & $3 \mathrm{~b}$ & 100 & $0.41 \pm 0.02^{*}$ & 54 \\
\hline 05 & $3 \mathrm{c}$ & 100 & $0.34 \pm 0.01^{*}$ & 62 \\
\hline 06 & $3 \mathrm{~d}$ & 100 & $0.57 \pm 0.05^{*}$ & 36 \\
\hline 07 & $3 \mathrm{e}$ & 100 & $0.31 \pm 0.02^{*}$ & 65 \\
\hline 08 & $3 \mathrm{f}$ & 100 & $0.36 \pm 0.01^{*}$ & 60 \\
\hline 09 & $3 \mathrm{~g}$ & 100 & $0.44 \pm 0.05^{*}$ & 51 \\
\hline 10 & $3 \mathrm{~h}$ & 100 & $0.39 \pm 0.02^{*}$ & 56 \\
\hline
\end{tabular}

Values are expressed as mean $\pm \operatorname{SEM}(\mathrm{n}=5)$

$* \mathrm{P}<0.001$ when compared to control group 


\section{RESULTS AND DISCUSSION}

All the compounds were synthesized by both conventional heating and microwave irradiation method. The conventional heating method is very time consuming while microwave irradiation method gives comparable yield with shorter reaction time. Though the reaction yields with MW irradiation were low there is no increase in yield observed after prolonged MW irradiation. All the compounds were characterized for IR and ${ }^{1} \mathrm{H}$ NMR spectral analysis and spectral data for compounds $\mathbf{3 a}-\mathbf{h}$ is given in table No-3. IR spectra of the compounds 3a-h exhibited an absorption band in the region of $3400 \mathrm{~cm}^{-1}$ due to $\mathrm{NH}$ and strong band in the region of $1700 \mathrm{~cm}^{-1}$ due to ester carbonyl. The ${ }^{1} \mathrm{H}$ NMR spectral data revealed a deuterium exchangeable broad singlet at $\delta$ 1.6-1.7 due to $\mathrm{NH}$ proton. All the new compounds have been screened for anti-inflammatory activity. Some of the compounds showed considerably antiinflammatory activity compared to diclofenac sodium.

Substituted aromatic amines gives better reaction yields as compared to aniline. The para substituted aromatic amines gives the highest yield compared to ortho and bisubstituted aromatic amine. While the compound $3 \mathrm{e}$ contain the bisubstituted aromatic amine moiety that gives the highest anti-inflammatory activity amongst all compounds synthesized.

\section{CONCLUSION}

In conclusion, we have described a highly efficient microwave-induced procedure for the preparation of Ethyl 3-arylaminobenzofuran-2-carboxylates from Ethyl -3-hydroxybenzofuran-2-carboxylate. This occurs remarkably fast, under mild condition, using inexpensive reagents and a household microwave oven as the irradiation source. The advantages of this microwave induced environmentally benign and safe protocol include a simple reaction set-up, application of commercially available reagents and catalyst with short reaction time.

\section{ACKNOWLEDGEMENT}

Authors are thankful to Dr.S.Ramchandra Setty, Principal, S.C.S.College of Pharmacy and Mr. R.N.Patil Principal I/C, SVPM's College of Pharmacy, Malegaon BkII, Baramati for providing laboratory facilities.

\section{REFERENCES}

1. (a) S. Caddick, Tetrahedron. 51, 10403, (1995). (b) S. Galema, Chem. Soc. Rev. 26, 233, (1997). (c) L. Parrcux, A. Loupy, Tetrahedron. 57, 9199, (2001).

2. (a) A. Loupy, A. Petit, J. Hamelien, B. F. Fexier, P. Jacquault, D. Mathe, Synthesis, 1213, (1988). (b) R. S. Verma, Green. Chem. 43, (1999).

3. C. H. Jyoti, R. N. Satheesha, K. Balkrishna, J. Chem. Sci. 119(4), 299, (2007).

4. C.W. Winder, J. Wax, M. Welford, J. Pharmacol. Exp. Ther. 148, (1965).
5. C.W. Winder, J. Wax, L. Serrano, E.M. Jones, M.L. Mcphee, Arthritis Rhem. 6, 36, (1963).

6. C. W. Winder, J.Wax, Scotti, R. A. Scherrer, E. M. Jones, F. W. Short, J. Pharmacol. Exp. Ther.138, 405, (1962).

7. R. Preeti, V. K. Srivastava, A. Kumar, Indian. J. Chem. 42B, 1729, (2003).

8. J. S. Kaltenbronn, R. A. Scherrer, F. W. Shork, E. M. Jones, H.R. Beatty, M. M. Saka, C. V. Winder, J. Wax, W. R. N. Williamson, Arzeim Forsch / Drug Res. 33, 621, (1983).

9. J. J. Perumattam, Chem Abstr, 124(15) 201802r, (1996).

10. S. Sharma, V. K. Srivastava, A. Kumar, Eur. J. Med. Chem. 37, 689, (2002).

11. A. Kumar, B. P. Jaju, J. N. Sinha, Indian. J. Pharm. Sci., 52(6), 257 , (1990).

12. K. M. Abou, S. Lashine, E. S. Abdulla, T. N. Abou, M.Z. Amer, J. Pharm. Sci. 2(1), 140, (1993).

13. D. H. Boschelli, D. T. Connor, D. A. Bornemeier, R. D. Dyer, J. A. Kennedy, P. J. Kuipers, G. C. Okonkwo, D. J. Schrier, C. D. Wright, J. Med. Chem. 36, 1802, (1993).

14. B. Goel, T. Ram, R. Tyagi, A. Bansal, A. Kumar, D. Mukherjee, et al, Eur. J. Med. Chem. 34(3), 544, (1999).

15. S. B. Mahajan, S. S. Sangapure, Y. S. Agasimundin, Curr. Sci. 45, 722, (1976).

16. S. Banerjee, K. S. Tapas, S. Mandal, D. P.Chandra, S. Sikdar, Inadian. J. Pharmacol. 32, 21, (2000).

17. C. A. Winter, E. A. Risely, C. W. Nuss, Proc. Soc. Exp. Med. 111, 544 , (1962).

18. T. A. Al-Howiriny, M. O. Al-Sohaibani, K. H. El-Tahir, S. J. Rafatullah, Natl. Remedies. 3/1, 54, (2003). 